\title{
ACUTE INFLAMMATORY RESPONSE
}

$I^{\mathrm{N}}$ Docember 1963, the New York Academy of Sciences held a conferonce on "The Acute Inflammatory Response", definod as the reaction to injury of the living mierocirculation and its contents. The past decade has seen much growth of interest in the inflammatory reaction, due partly to the introduction of electron microscopy and partly to widespread intorest in immunological responses which provoke inflammation.

The published Proceedings* open with an account of the electron microscopy of inflamed vessels. It was the work of Majno and Palade which demonstrated unequivocally that colloidal particles such as plasma protein leave inflamed vessels by traversing gaps between the endo. thelial cells. These gaps are induced to appear by the action of all substances known to increase vascular permeability to protein. In the case of these substances the action is confined to venules. In true inflammation, both venules and capillaries are involved, their relative participation varying with the type of injury.

The next communication is by $\mathbf{E}$. M. Landis, who investigated and filmed the passage of free- or proteinbound dye through normal capillaries and venules. He found that free dye left the circulation too rapidly to be consistent with transport through a vacuole system as at present envisaged. Passage of protein-bound dye was 'spotty', indicating the presence of a set of 'pores' larger in size and less numerous than those permitting the escape of free dye.

Next follows an account of the electron microscopy of leucocyte emigration from inflamed vessels. The white cells were found to penetrate the endothelium by extending a pseudopod into the inter-endothelial junction. Monocytes left in similar fashion, but lymphocytes were not seen to leave the vessels. Diapedesis of red cells occurred through open inter-endothelial junctions. A similar electron microscopic study of high quality was described in relation to vascular regeneration after injury. These observations supported the existing viow of budding from adjoining surviving vessels. A study of lymphatics completes the ultra-structural section. It would seem that lymphatics behave essentially in similar fashion to venules but that the 'porosity' of their inter-endothelial junctions is normally greater.

The next main section of the volume is devoted to the problem of endogenous chemical mediators of inflammatory change. Since the era of Menkin, a number of potential mediators have been discovered, notably bradykinin and similar peptides (kinins), the kinin-forming onzymes and

* Annals of the New York Academy of Sciences. 116, Article 3: The Acute Inflammatory Response, by W. G. Spector, Albert S. Gordon and 39 othe 6 dollars. the globulin permeability factor. Bradykinin has actually been purified and synthesized. Nevertheless, in contrast with the chemical success, G. P. Lewis was unable to report unequivocal evidence for active participation of this peptide in bringing about the inflammatory reaction. The same is true for the enzymes which form it (kininogenases such as kallikrein) and for the globulin permea. bility factor. Novertheless, in all three cases, suggestive pointers have been found, but investigators badly need specific antagonists of these substances as experimental tools. At present such inhibitors are not forthcoming. The complexity of the inter-relationships between the factors and also similar plasma protein components of the blood coagulation system is, however, alroady painfully apparent.

In contrast with the confusion over the role of high molecular weight substances, there is now general agreement on the part played by histamine. This substance is realized to be generally a minor factor in full-blown inflammatory reactions, although often responsible for the phase of vascular change which occurs immediately after injury. A dissident voice is raised by Schayer, who believes that augmented histamine synthesis often maintains the later stages of the response. Serotonin (5-HT) is now thought to be a mediator only of certain specialized reactions in the rat. 'Anti-inflammatory' amines, for example adrenaline, appear also to be involved in inflammation in that enhanced activity of the enzymes which destroy them seems to contribute to the development of the reaction.

'The conference brought forth one 'new' potential mediator, a factor or factors present in lymph node extracts. The relationship of this active principle to the known activity on blood vessels of tissue extracts in general remains to be elucidated.

The third section of the volume is devoted to leucocyte emigration. One point of special interest is the clear evidence that loss of plasma protein and diapedesis of leucocytes from inflamed vessels are separate processes. At any one time any one vossel may be leaking protein or permitting the escape of leucocytes quite independently. Another interesting feature of the symposium is a discussion of adhesion of leucocytes to inflamed endothelium as a phenomenon of surface charge, invoking the behaviour of lyophobic colloids as an analogy.

The final quarter of the publication provides a number of précis reviews of topics closely relevant to the main theme, for example, hypersensitivity reactions, the role of the nervous system and of eosinophils. Looking back over the past ten years, and in spite of the problems which remain unresolved, one can only be impressed by the progress made in this field.

\section{ULTRA-WEAK EMISSION IN THE VISIBLE AND ULTRA-VIOLET REGIONS IN OXIDATION OF SOLUTIONS OF GLYCINE BY HYDROGEN PEROXIDE}

\author{
By Dr. A. A. GURWITSCH, V. F. EREMEYEV and Yu. A. KARABCHIEVSKY \\ Cabinet of Mitogenesis, Institute of Normal and Pathological Physiology, USSR Academy of Medical Sciences
}

$\mathrm{M}$ OST chemiluminescent reactions proceeding in vitro involve the participation of molecular oxygen, or peroxides which readily release molecular oxygen. At the present time a broad. study is being made (by photomultiplier recording) of the chemiluminescence of these processes in the visible region of the spectrum ${ }^{1-5}$. The aim of the present work is to determine whether or not there is an ultra-violet component in this emission.

Photomultiplier investigations of this aspect of the phenomenon were particularly interesting, because a 